BABELAO 5 (2016), p. 117-125

(C) ABELAO (Belgium)

\title{
Le livre des Actes dans la Bible éthiopienne. A propos d'un livre récent
}

Par

\section{Jean-Louis Simonet}

Université catholique de Louvain, Louvain-la-Neuve

T es éditions Picwick Publications (Eugene, Oregon, USA) ont publié, en octobre 2014, dans la série Ethiopic Manuscripts, Texts, and Studies, vol. 19, une édition critique du texte éthiopien $\left(\mathrm{Ge}^{6} \mathrm{ez}\right)$ des Actes des Apôtres, que nous devons à L. Curt Niccum, professeur associé de Nouveau Testament à 1'Abilene Christian University, et codirecteur du projet «Textual History of the Ethiopic New Testament $»$. Niccum développe ainsi la thèse de doctorat qu'il avait présentée en 2000 à l'Université Notre Dame'

Cet ouvrage est le deuxième fruit accessible au public d'un projet nommé "International Project on the Text of Acts»

\footnotetext{
${ }^{1}$ L.C. NiCCUM, The Bible in Ethiopia. The Book of Acts (Ethiopic Manuscripts, Texts and Studies Series, 19), Eugene, 2014, p. xii, 354.
} 
(I.P.T.A), initié par C.D. Osburn ${ }^{2}$ et Th.C. Geer, et repris entretemps par l'Institut für neutestamentliche Textforschung de Münster. Ce projet prévoit l'édition d'un texte critique des Actes en grec, latin, syriaque, copte, arménien (dejà paru), géorgien et éthiopien.

Les éditions Picwick Publications et la série Ethiopic Manuscripts, Texts, and Studies étant encore relativement peu connues $^{3}$, il nous a paru d'autant plus important de recenser cet ouvrage, qui risquait de rester inaperçu, malgré son caractère remarquable.

\section{Présentation de l'ouvrage}

L'ouvrage comporte 88 pages d'introduction, 179 pages de texte et d'apparat critique, 3 appendices, une bibliographie, un index des auteurs et un index des matières mentionnées.

Le premier chapitre de l'introduction est consacré à l'histoire de la transmission du texte des Actes en éthiopien. Après une brève discussion des origines de la Bible éthiopienne, Niccum en vient à un historique des recherches. H. Zotenberg est le premier à distinguer deux groupes de manuscrits (les familles $\mathrm{A}$ et $\mathrm{B}$ de Niccum). I. Guidi est le premier à remarquer que la famille $\mathrm{B}$ provient d'une révision de la Bible éthiopienne sur une traduction arabe, la famille A représentant, par contre, le texte antérieur à cette révision. A partir de 10 chapitres de Matthieu, L. Hackspill conclut que la Bible éthiopienne a été traduite, vers 500, d'un manuscrit grec «syrooccidental ». Hackspill voulait ainsi, utilisant la terminologie élaborée par Westcott et Hort, parler de ce que nous appelons aujourd'hui le texte byzantin. Mais plusieurs, ignorant le sens de cette terminologie, ont pensé à rechercher une part d'influence du syriaque dans la Bible éthiopienne. A. Vööbus, tout en notant de fortes traces d'influences du grec et de l'arabe, affirme, exemples à l'appui, que la Bible éthiopienne est traduite de la Vetus Syra, mais a du être soumise à plusieurs révisions ultérieures. R. Zuurmond, travaillant avec des manuscrits plus nombreux et plus anciens que ceux connus par Vööbus, a montré que les strates les plus anciennes de la version éthiopienne ne montrent qu'une influence grecque, et que les exemples d'influence syriaque cités par Vööbus proviennent de développements ultérieurs dans la transmission du texte éthiopien (en fait il n'y a influence du syriaque - y compris parfois

${ }^{2}$ C.D. Osburn mentionne ce projet dans son article «The Search for the Original Text of Acts - The International Project on the Text of Acts », JSNT 44 (1991), p. 39-55.

${ }^{3}$ La série «Ethiopic Manuscripts, Texts and Studies », dirigée par St. Delamarter, compte déjà quatre volumes parus, mentionnés, immédiatement après le titre, dans l'ouvrage que nous recensons ici. 14 volumes au moins sont donc en préparation. 
de la Vetus Syra - qu'à travers la traduction arabe faite sur le syriaque qui a été utilisée par les réviseurs qui sont à l'origine de la famille B). M.-É. Boismard et A. Lamouille pensent que la Bible éthiopienne est traduite du grec, mais à partir du texte « occidental», un texte court dont l'éthiopien originel serait le seul témoin relativement pur. Malheureusement, ce texte originel n'est préservé qu'en partie, car la version éthiopienne aurait subi deux révisions indépendantes, l'une sur le texte grec « alexandrin » (la famille A), l'autre sur la Peshitta syriaque (la famille B). Cette théorie bute sur le fait que le premier de ces réviseurs omet de combler les leçons ultra-brèves des ch. 27 et 28 des Actes. En outre, les traces d'une Vorlage arabe pour la famille B sont claires et il est incompréhensible que Boismard et Lamouille ne les aient pas vues ${ }^{4}$.

La question de la date de la traduction éthiopienne est également discutée tout au long de l'examen de ces multiples théories. Niccum la situe quelque part entre 350 (établissement ferme de l'Eglise éthiopienne) et 525 (les inscriptions du roi Caleb, contenant des citations bibliques), avec une préférence pour la seconde moitié du $\mathrm{IV}^{\mathrm{e}}$ siècle. Cependant, certains détails (énumérés à la p. 13) pourraient indiquer un certain intervalle entre la traduction des Evangiles et des Epîtres pauliniennes, d'une part, les Actes et les Epîtres catholiques de l'autre, mais ces détails pourraient aussi être interprétés comme des harmonisations ultérieures. Le problème, pour les Actes, c'est que nous n'avons aucun manuscrit antérieur au XIV ${ }^{\mathrm{e}}$ siècle ${ }^{5}$; les plus anciennes citations des Actes, dans le Kebra Nagast, ne remontent qu'au XIII ${ }^{\mathrm{e}}$ siècle. Et même si certains détails montrent une fidélité d'ensemble de la famille A par rapport à la traduction originelle, d'autres détails montrent que le texte éthiopien des Actes a connu un certain développement, difficile à mesurer, entre la traduction primitive et ses plus anciens témoins ${ }^{6}$.

\footnotetext{
${ }^{4}$ En apprenant l'éthiopien à Louvain-la-Neuve, avec le Père U. Zanetti comme professeur, nous avons étudié Act 27 et 28 selon le manuscrit eth 42 de la Bibliothèque Nationale de France. Même dans ce manuscrit, qui appartient à la famille $\mathrm{A}$, les traces d'influence arabe sont évidentes, surtout dans les noms propres. Nous avons pris soin de signaler nos conclusions par courriel à M.-É. Boismard, lequel les a rejetées en bloc, se contenant de me renvoyer (ne varietur) à ses écrits.

${ }^{5}$ Une grande partie de l'Ethiopie a été occupée, de 1531 à 1543, par un émirat islamique qui a procédé à une destruction systématique des Eglises, des monastères, et de leurs manuscrits, et à un massacre des prêtres et religieux, accompagnés d'une tentative d'islamisation forcée de la population. Nil novi sub sole...

${ }^{6}$ Niccum ne dispose que de 6 manuscrits plus ou moins complets comme témoins de la famille A (voir liste p. 75-84), mais presque tous souffrent de négligences scribales, voire d'un début de révision sur l'arabe. Il peut aussi recourir au témoignage de 14 lectionnaires éthiopiens, dont le
} 
Niccum regroupe un nombre important mais non-homogène de manuscrits sous le label $\mathrm{Ab}$, une appellation justifiée par le fait que ces manuscrits sont encore proches de la famille A, tout en préfigurant le texte de la famille B. Dans ce groupe, la révision sur l'arabe n'est que partielle. L'absence d'homogénéité indique que le processus de révision s'est fait sans direction d'ensemble, du XIII ${ }^{\mathrm{e}}$ au $\mathrm{XIV}^{\mathrm{e}}$ siècle. Le témoin le plus ancien de ce groupe date de 1400 .

La famille $\mathrm{B}$, par contre, est très homogène, ce qui témoigne d'un effort concerté de révision sur l'arabe, au $X V^{\mathrm{e}}$ ou au début du XVI ${ }^{\mathrm{e}}$ siècle (le plus ancien manuscrit de cette famille est du $\mathrm{XVI}^{\mathrm{e}}$ siècle).

Le deuxième chapitre est consacré à la fidélité du texte de la famille A par rapport à son archétype grec. Niccum relève le fait qu'en certains endroits l'éthiopien a transcrit une désinence d'un cas grec, ou translittéré des mots grecs; parfois l'éthiopien atteste une variante qui n'est connue qu'en grec, ou encore, manifeste une erreur de traduction qui ne s'explique qu'à partir du grec. Niccum examine ensuite les rapports possibles entre l'éthiopien et les principales versions anciennes des Actes. Les versions arabes ne sont utiles que pour comprendre l'origine de la famille B, que Niccum voit comme proche du texte du manuscrit arabe 151 du Sinaï et de celui de la colonne arabe de la tétraglotte de Milan. L'exemple le plus probant est le fait que la finale des Actes, dans la famille B, traduit littéralement une note marginale du Sinaï arabe 151 et d'autres témoins arabes. Les versions latines, syriaques et coptes n'offrent par contre aucun parallèle qui ne pourrait être compris qu'en termes de dépendance littéraire de l'éthiopien. Ce chapitre se termine par une table des 221 noms propres des Actes, en éthiopien, grec, syriaque et copte.

Dans le troisième chapitre, Niccum examine quel type de texte grec a pu être utilisé par les rédacteurs de la traduction éthiopienne. L'auteur utilise plusieurs méthodes, en prenant soigneusement en compte les limitations de la langue éthiopienne pour rendre le grec. Il commence en comparant l'éthiopien avec les passages variants étudiés par K. ALAND et al. dans Text und Textwert der Griechischen Hanschriften des Neuen Testaments, III. Apostelgeschichte (Berlin-New-York: Walter De Gruyter, 1993); puis il utilise l'analyse quantitative de Colwell et Tune, et finalement, la Comprehensive Profile Method de B. Ehrman. Le texte éthiopien des Actes appartient indiscutablement à la famille alexandrine. Les variantes occi-

plus ancien date du $\mathrm{XIV}^{\mathrm{e}}$ siècle, mais ces lectionnaires n'offrent qu'une partie du texte des Actes, plus ou moins restreinte en fonction du nombre des lectures bibliques retenues. Il est intéressant d'observer qu'en éthiopien également, les lectionnaires donnent accès à une strate plus ancienne de la transmission du texte biblique. 
dentales sont rares, et l'influence du texte byzantin, bien que plus constante, est cependant limitée. Niccum prend encore la peine d'examiner (p. 61-67) 23 endroits où l'éthiopien semble présenter des variantes peu attestées. Ces variations, conclut-il, sont souvent apparentes ou accidentelles. Il reste néanmoins 2 accords avec $\mathrm{p}^{74}$ et deux avec 05 (D), et 6 accords possibles dont 3 avec 05 (D), 1 avec $p^{45}, 1$ avec 08 (E), et 1 avec 1739 et 1891.

Le quatrième chapitre présente l'édition critique et son apparat. Niccum commence en rappelant les lacunes bien connues des deux éditions précédentes, celles de Rome (en 1548-1549) et celle de Th.P. Platt (1830). La première part du manuscrit 23 de la Bibliothèque du Vatican, un manuscrit de la famille $\mathrm{A}$, mais incomplet (les passages manquants étant traduits en éthiopien à partir de la Vulgate !); la seconde part d'un seul manuscrit $^{7}$, appartenant à la famille $\mathrm{B}$, avec des corrections occasionnelles sur l'édition de Rome. L'édition de Niccum est la première édition critique $\mathrm{du}$ texte éthiopien le plus ancien tel qu'accessible par les manuscrits (mais pas d'un texte original éthiopien que l'on pourrait parfois tenter de reconstruire par émendation conjecturale). Elle suit généralement le manuscrit 20 de l'Ambrosiana de Milan, le témoin le plus ancien de la famille A, mais en le corrigeant quand il y a lieu à l'aide des autres témoins de la même famille.

L'apparat critique est introduit (p. 73-74) par une liste de sigles parfois inhabituels, mais bien expliqués, et nous ne saurions trop recommander au lecteur de bien s'imprégner du sens de ces sigles avant de commencer la lecture de l'apparat. L'auteur explique les principes qui ont présidé au choix des variantes retenues dans l'apparat critique, forcément incomplet pour éviter la surcharge; il précise notamment que toutes les variantes attestées avant le $\mathrm{XVII}^{\mathrm{e}}$ siècle y sont inclues. Enfin, Niccum fournit une liste de 117 manuscrits continus et de 17 lectionnaires ou textes liturgiques avec leur localisation, et en donnant, autant qu'il en soit informé, le contenu biblique, la date et l'appartenance à une famille textuelle ${ }^{8}$. Niccum précise

\footnotetext{
${ }^{7}$ Th. Pell-Platt ne précise pas quel manuscrit il a utilisé. Il s'agit sans doute d'un manuscrit de la British Library à Londres, mais personne ne semble savoir précisément lequel (voir à ce sujet $\mathrm{Br}$. M. METZGER, The Early Versions of the New Testament, Oxford: Clarendon Press, 1977), p. 231, en particulier la n. 1).

${ }^{8} \mathrm{Il}$ n'y a pas que les familles $\mathrm{A}, \mathrm{B}$ et $\mathrm{Ab}$, que nous avons déjà pu présenter. Un manuscrit unique présente une conflation du texte de la famille $\mathrm{Ab}$ avec une nouvelle traduction faite sur base d'un modèle arabe. Pour certains manuscrits de la famille B, des sous-groupes ont pu être établis; en outre, d'autres manuscrits de cette famille peuvent être qualifiés de « faibles » - ce qui laisse entendre, nous le supposons, que des leçons de A ou de Ab subsistent en certains endroits ; ici encore des sous-groupes apparaissent. Enfin,
} 
que la liste devrait encore s'accroître, au fur et à mesure qu'est révélée l'existence de manuscrits encore inconnus. Un chiffre entre parenthèse indique le sigle qui désigne le manuscrit dans l'apparat. 18 manuscrits continus sont cités constamment dans l'apparat, et 20 autres sont cités dans 243 versets contenant des variations génétiques importantes pour l'histoire du texte (voir listes en p. 84), et les 17 lectionnaires ou livres liturgiques sont tous cités dans l'apparat. Les éditions de Rome et de Pell-Platt sont aussi citées, sauf quand l'édition de Rome traduit la Vulgate, et sauf quand Pell-Platt est influencé par l'édition de Rome.

L'appendice A (p. 269-271) contient un supplément de lectures fautives de l'éthiopien qui suggèrent une Vorlage grecque. L'appendice B (p. 272-277) est une table des accords entre la famille $\mathrm{A}$ du texte éthiopien et différents témoins des Actes en 419 lieux variants. Cette table permet d'établir un ordre décroissant de proximité. Les témoins les plus proches sont $\mathrm{p}^{45}$, puis $\mathrm{B}$, boh, $\boldsymbol{\kappa}, \mathrm{p}^{74}, 1175, \mathrm{~A}$ et vg (allant de 86 à $82 \%$ d'accords). Last but not least, l'appendice C (p. 278-330) contient une liste de variantes du Nouveau Testament grec qui bénéficient du soutien apparent de la famille A du texte éthiopien. Le texte éthiopien cité est traduit en anglais, puis l'auteur donne une liste de témoins non éthiopiens qui attestent la même variante; des notes de bas de page discutent du degré de probabilité du soutien d'une variante par l'éthiopien, lorsqu'il y a lieu de le faire. Cet appendice sera très utile aux critiques textuels, surtout s'ils ne sont pas eux-mêmes éthiopisants.

\section{Appréciation}

Ce nouvel ouvrage comble un grand vide, et C. Niccum maîtrise admirablement bien son sujet. L'ouvrage est dans l'ensemble bien présenté, clair et est un modèle à suivre en matière d'édition d'une version ancienne d'un livre biblique. L'histoire textuelle des Actes éthiopiens est établie clairement, et les théories erronées de Vööbus et de Boismard sont, espérons-le, écartées définitivement. Le souci de rendre l'ouvrage accessible même au lecteur qui ignorerait le ge'ez est d'autant plus louable qu'il est rare dans des publications de ce type. Un défaut mineur toutefois dans la présentation: les chiffres indiquant le début des versets bibliques sont en petits caractères, assez difficiles à repérer dans le texte.

Peut-on attendre de l'avenir de nouveaux progrès dans l'édition des Actes éthiopiens ? Ce qui suit ne dépend pas de Niccum, qui souligne lui-même cette lacune; mais on peut regretter, en tout cas, pour mieux comprendre l'origine des fa- 
milles $\mathrm{Ab}$ et $\mathrm{B}$, l'absence d'une recherche portant sur l'ensemble des manuscrits arabes des Actes, et, plus particulièrement, l'absence d'une édition critique de la version melkite $\mathrm{du} \mathrm{IX}^{\mathrm{e}}$ siècle (une révision du Sinaï arabe 151). Cette absence est toutefois partiellement compensée par le fait qu'un manuscrit tardif de cette version a été édité par Th. Erpenius ${ }^{9}$ à Leyde en 1616 - C. Niccum ne mentionne pas cette édition. Il ne semble pas non plus avoir comparé le texte éthiopien avec la colonne arabe de la Polyglotte de B. Walton (Londres, 1657) ${ }^{10}$, laquelle édite un manuscrit de la version melkite du XIII ${ }^{\mathrm{e}}$ siècle, faite sur le grec.

Ce retard dans l'étude des Actes en arabe fait que nous sommes incapables de voir si la ou les révisions éthiopiennes menant aux familles $\mathrm{Ab}$ et $\mathrm{B}$ découlent du même type de texte arabe, ou de plusieurs textes arabes distincts; il empêche aussi une évaluation sérieuse de la technique de traduction utilisée par les réviseurs éthiopiens.

La liste établie par Niccum des manuscrits éthiopiens des Actes souffre de quelques lacunes - nous parlons ici de manuscrits connus depuis longtemps. Le manuscrit Tanasee 12 de Hambourg est omis. Il date du XVII ${ }^{\mathrm{e}}$ siècle, et nous déduisons de ce qu'en disent Boismard et Lamouille qu'il devrait appartenir à la famille $\mathrm{B}$. Son absence est donc sans doute sans (ou de peu d') importance pour la reconstitution du texte le plus anciennement accessible de la famille A. Un peu plus significative, par contre, pourrait être l'omission des deux lectionnaires suivants: Paris, B.N. Eth. 43, fol. 67, XV $\mathrm{XV}^{\mathrm{e}}$ s., contenant Act 13:27-34, et Paris, B.N. Eth. 44, fol. 8v-9v, XVI s., contenant Act 1:15-22. Ces deux fragments, que nous avons collationnés, appartiennent à la famille A. Enfin, en compulsant le catalogue de W. Macomber ${ }^{11}$, nous constatons l'omission du lectionnaire EMML 4752, provenant de Warana Baro $\left(\mathrm{XV}^{\mathrm{e}} \mathrm{s}\right.$.) et contenant Act 1:15-20, 2:22-33, 3:12-4:4, 8:26-40, 18:11-31, ainsi que celle de EMML $2197\left(\mathrm{XVI}^{\mathrm{e}}-\mathrm{XVII}{ }^{\mathrm{e}}\right.$ s.), et d'autres manuscrits ou lectionnaires plus récents. N'ayant pas vu ces documents, nous ne pouvons évaluer la portée de ces lacunes.

\footnotetext{
${ }^{9}$ Novum Domini nostri Iesu Christi Testamentum Arabice ex Bibliotheca Leidensi.

${ }^{10}$ Biblia Polyglotta (6 tomes).

${ }^{11}$ Catalogue of Ethiopian Manuscripts Microfilmed for the Ethiopian Manuscript Microfilm Library, Addis Abeba, and for the Monastic Manuscript Microfilm Library (EMML), Collegeville (Collegeville, 56321 Minnesota: Monastic Manuscript Microfilm Library of St John's Abbey and University; vol. I (mss 1-300), 1975; vol. II (mss 301-700), 1976; vol. III (mss 701-1100), 1978; vol. IV (mss. 1101-1500), 1979; vol. V (mss. 1501-2000), 1981; vol. VI (mss. 2001-2500), 1982; vol. VII (mss. 2501-3000), 1983; vol. VIII (mss. 3001-3500), 1985; vol. IX (mss. 3501-4000), 1987; vol. X (mss. 4001-5000), 1993. Ce catalogue est encore incomplet.
} 
C. Niccum n'a pas inclus dans son apparat critique les variantes des manuscrits 526, 529, 530 et 531 de la British Library, lesquels figurent dans l'apparat critique de Boismard et Lamouille. Il fallait certes bien limiter la taille de l'apparat critique, mais c'est toujours avec regret que nous voyons des témoins cités dans une étude antérieure ne pas être repris dans une édition qui se veut définitive.

Nous avons collationné pour les chapitres 1 à 10 des Actes, le manuscrit 518 (du XVIII ${ }^{\mathrm{e}}$ s.) de la Bibliothèque des Bollandistes de Bruxelles, qui ne figure pas dans l'apparat critique de Niccum. Ce dernier classe, erronément, ce manuscrit 518 comme porteur d'un texte de la famille B, alors que le nombre de leçons A qu'il soutient devrait plutôt le faire classer comme appartenant à la famille $\mathrm{Ab}$. Il faut espérer qu'une telle erreur soit unique, mais qui va vérifier pour tous les autres manuscrits laissés de côté par l'apparat? Ce manuscrit porte aussi un assez grand nombre de leçons uniques ou peu attestées à notre connaissance, ce qui pourrait montrer qu'une étude plus détaillée et complète de l'histoire du texte éthiopien pourrait encore être porteuse d'enseignements.

Les images les plus récemment créées de manuscrits éthiopiens en possession de la Hill Monastic Manuscript Library ne révélant que des manuscrits récents, il semble peu probable que les bibliothèques éthiopiennes recèlent encore des manuscrits anciens qui pourraient être importants pour mieux établir l'histoire du texte éthiopien des Actes $^{12}$. Par contre, des découvertes pourraient toujours être possibles chez quelques antiquaires et dans les collections privées telles que celle où nous avons eu le privilège de découvrir le manuscrit 2345, le plus ancien témoin de la famille $\mathrm{Ab}$.

La principale faiblesse du livre de Niccum est l'absence presque totale des citations bibliques faites par les auteurs éthiopiens, à l'exception des quelques citations du Kebra Nagast. Nous établissons, depuis des années, un répertoire de ces citations, grâce aux ressources de la Bibliothèque de l'Université de Louvain-la-Neuve. Nous pouvons ainsi affirmer la présence de citations des Actes chez au moins 23 auteurs éthiopiens ${ }^{13}$, à partir du XIV ${ }^{\mathrm{e}}$ siècle. En outre, il s'agit fréquemment de citations longues. Nous n'avons pas étudié ces citations en détail, mais il

\footnotetext{
${ }^{12}$ A l'exception probable du « vieux manuscrit brûlé » $\left(\mathrm{du} \mathrm{XV}^{\mathrm{e}}\right.$ siècle ?) vu naguère à Rome par R. Zuurmond, dans une petite bibliothèque dont il n'a pas conservé l'identification (cf. p. 74, n. 16). Si quelque lecteur possédait une information permettant de localiser ce manuscrit, nous serions heureux qu'il le fasse savoir.

${ }^{13}$ Nous ne parlons ici que d'œuvres éthiopiennes originales, sans inclure les citations contenues dans des œuvres de traduction (les Ethiopiens ont traduit un nombre important d'ouvrages grecs, coptes, syriaques, arabes et même latins).
} 
est facile de voir que nombre d'entre elles attestent le texte de la famille A. Nous pensons ici en particulier aux citations des œuvres de Giyorgis de Saglā et du Négus Zar'a Yā'qob, deux auteurs du $\mathrm{XV}^{\mathrm{e}}$ siècle, lesquels citent forcément des manuscrits plus anciens. Si quelque progrès reste à réaliser pour mieux rétablir l'état accessible le plus ancien des Actes des Apôtres, ce sera vraisemblablement essentiellement cette voie qu'il faudra suivre. Peut-être un jour publierons-nous une étude de ces citations, si Dieu nous prête vie, et si personne d'autre ne s'en charge avant nous (nous prêterions volontiers notre concours à un jeune chercheur qui voudrait s'engager dans cette voie).

Précisons enfin que l'éloge que C. Niccum fait de nous («a constant conversation partner $»)$ dans ses Acknowledgments, $p$. ix, nous paraît aimable mais quelque peu exagéré. C. Niccum nous avait envoyé, il y a déjà longtemps, un brouillon de son édition, et nous lui avons transmis des remarques sans doute utiles, mais jusqu'au chapitre 7 des Actes seulement; par la suite, depuis des années, nous avons cessé de le faire, à cause de notre implication grandissante dans la recherche sur les Actes arméniens.

Ceci dit, nous souhaitons que l'ouvrage remarquable que nous recensons ici jouisse de la plus grande diffusion, et le recommandons vivement à tout chercheur qui s'intéresse à l'histoire du texte des Actes des Apôtres ou à la Bible éthiopienne, faisant nôtre cette adaptation par Niccum (p. 68) d'une citation de Westcott et Hort: «Knowledge of versions ${ }^{14}$ should precede final judgment upon readings ».

${ }^{14}$ Westcott et Hort avaient dit « knowledge of manuscripts ». 
